\title{
Optimum swimming pathways of fish spawning migrations in rivers
}

\author{
Brandon McElroy, ${ }^{1}$ Aaron Delonay, and Robert Jacobson \\ U.S. Geological Survey, Columbia Environmental Research Center, 4200 New Haven Road, Columbia, Missouri 65201 USA
}

\begin{abstract}
Fishes that swim upstream in rivers to spawn must navigate complex fluvial velocity fields to arrive at their ultimate locations. One hypothesis with substantial implications is that fish traverse pathways that minimize their energy expenditure during migration. Here we present the methodological and theoretical developments necessary to test this and similar hypotheses. First, a cost function is derived for upstream migration that relates work done by a fish to swimming drag. The energetic cost scales with the cube of a fish's relative velocity integrated along its path. By normalizing to the energy requirements of holding a position in the slowest waters at the path's origin, a cost function is derived that depends only on the physical environment and not on specifics of individual fish. Then, as an example, we demonstrate the analysis of a migration pathway of a telemetrically tracked pallid sturgeon (Scaphirhynchus albus) in the Missouri River (USA). The actual pathway cost is lower than $10^{5}$ random paths through the surveyed reach and is consistent with the optimization hypothesis. The implication - subject to more extensive validation - is that reproductive success in managed rivers could be increased through manipulation of reservoir releases or channel morphology to increase abundance of lower-cost migration pathways.
\end{abstract}

Key words: energetics; energy expenditures; least cost; least-cost migratory pathways; fluvial velocity fields; migration; Missouri River, USA; pallid sturgeon; Scaphirynchus albus; spawning migration.

\section{INTRODUCTION}

Many fish species that spawn in fluvial habitats migrate long distances upstream. To the degree that they use the same resources to swim and to develop gametes, there is a clear evolutionary advantage to traversing a migratory pathway that minimizes energy expenditure. Specifically, as has been demonstrated for brown trout (Salmo trutta), chinook salmon (Oncorhyncus tshawytscha), and sockeye salmon (Oncorhynchus nerka), the fecundity of spawning fishes decreases with migratory distance and difficulty (Kinnison et al. 2001, 2003, Crossin et al. 2004, Jonsson and Jonsson 2006). While the migratory cost in the study of Kinnison and others (2001) is singularly a function of total migration distance, in large rivers with a great variety of possible paths through a range of water velocities, the cost should be a function of the total path distance and velocities experienced along it. This assumes that there are sufficient cues for fish to start migrating and that migration is its primary behavior. In this paper we develop the theoretical framework for analyzing leastcost migratory pathways of fluvial fish including a cost function for comparing pathways, and apply it to an example of telemetry-based migration pathway data for

Manuscript received 15 June 2011; revised 24 August 2011; accepted 1 September 2011. Corresponding Editor: S. J. Simpson.

${ }^{1}$ Present address: University of Wyoming, Department of Geology and Geophysics, Department 3006, Laramie, Wyoming, USA. E-mail: bmcelroy@uwyo.edu the endangered pallid sturgeon (Scaphirhynchus albus) in the Lower Missouri River, USA.

Pallid sturgeon is a federally listed, endangered species endemic to the Mississippi Basin (Mayden and Kuhajda, 1997). In spring reproductive females migrate distances of $100-150 \mathrm{~km}$ or more (DeLonay et al. 2009) to spawn upstream. Because flows in the Missouri River are highly managed for purposes including navigation, flood control, power generation, and ecosystem needs, the effects of reservoir releases and associated flow velocities during migration and spawning periods could have implications for successful reproduction of pallid sturgeon. Moreover, channelization for navigation has substantially altered the river's velocity distribution along its lower $1200 \mathrm{~km}$ (Jacobson and Galat 2006). Within this context it has been observed that adult pallid sturgeon utilize relatively slow-velocity regions along the inner banks of bends during migration (DeLonay et al. 2010). Possibly these fish are selecting pathways in order to optimize their migration. For these reasons, we seek to elucidate the physical controls on energy expenditure of fishes during spawning migrations in rivers.

The general problem of finding a most efficient or otherwise optimal path from a starting location to an ultimate location or series of locations is well developed in the context of networks such as roadways, railways, electric transmission lines, the internet, or other features of physical infrastructure (Ahuja et al. 1993, Stoll 1989, Huitema 2000, Brandimarte and Zotteri 2007). Regular lattices such as a digital elevation models (DEM) can also be viewed as a series of interconnected locations (Rees 2004). Here, we employ a discretized three- 
dimensional flow field in a river as a regular lattice to represent a network of individual flow-volume elements, or "voxels." The goal is to determine an optimum manner by which fish traverse this generalized network. The two essential pieces of this problem are a mathematical description of a network, $X$, and a cost function $f(X)$ for its traversal. For Rees' case of footpaths over hills (2004), the network nodes are defined by the cells of the DEM and the cost function depends on the local rate of topographic change between cells. In the present application, the hydrodynamics of swimming fish provide a cost function, and flow velocities in the voxels are the network through which a fish must traverse a pathway. Using this context we develop a theory for discerning optimum pathways by which upstream-migrating fish might travel. The theory can be used to test the hypothesis that fish migrating upstream to spawn select pathways that minimize their energy expenditure.

\section{Theory of Energetic Cost for Fish Migrations IN RIVERS}

To develop a network cost function, we investigate the complex relation of swimming to energy expenditure of fish. With a data set including a variety of carangiform swimmers, Boisclair and Tang (1993) demonstrated that mass and speed together account for $80 \%$ of the variance of oxygen consumption, and therefore energetic cost, of fishes swimming against a unidirectional current. Their analysis (1993) does not account for differences between continuous, steady swimming and intermittent, burstand-glide swimming, which is theorized to save energy relative to steady swimming (Weihs 1974). Weihs (1974) postulates that relative swimming mode efficiency is related to size, to speed, and most importantly to how drag on a fish's body during undulatory swimming motions compares to drag on a straight-bodied, gliding fish. Despite the existence of empirical evidence that fish in conditions similar to those assumed here swim with a burst-and-glide strategy (e.g., Colavecchia et al. 1998, Hinch and Rand 2000) drag information for the two swimming stages is generally not available in sufficient detail, i.e., by species, size, and environmental conditions, to make its application possible. Therefore, the current theoretical development for large fish (e.g., adult pallid sturgeon, $\sim 1 \mathrm{~m}$ in length) moving at modest rates relative to the water around them (e.g., Missouri River, water velocity of $\sim 1-1.5 \mathrm{~m} / \mathrm{s}$ ) is necessarily limited to continuous swimming.

With this context we conduct a dimensional analysis of swimming drag in order to derive a cost function with which to compare the range of possible migratory pathways. The relevant Reynolds number for a typical fish under these conditions is $R_{\mathrm{f}}=U_{\mathrm{r}} L / v>10^{6}$ where $U_{\mathrm{r}}$ is the typical swimming velocity relative to the surrounding water $(>1 \mathrm{~m} / \mathrm{s}), L$ is typical fish length $(1 \mathrm{~m})$, and $v$ is the kinematic viscosity of water $\left(10^{-6} \mathrm{~m}^{2} / \mathrm{s}\right)$. Empirical evidence from Anderson and others (2001) strongly suggests that the boundary layer on a fish in these conditions will be fully turbulent. Additionally Drucker and Lauder (2002) show with experimental visualization the reverse von Karman vortex streets shed by swimming fish. These are responsible for thrust that powers a swimming fish and similarly are the reason the form drag is minimized around them. Therefore friction drag is assumed to be the dominant drag and form drag can be neglected with some minor errors likely accrued (see Anderson et al. 2001). The drag on a swimming fish can be written as

$$
D=\frac{1}{2} \rho C_{\mathrm{D}} S U_{\mathrm{r}}^{2}
$$

where $D$ is the total drag (in $\mathrm{kg} \cdot \mathrm{m} / \mathrm{s}^{2}$ ), $\rho$ is the fluid density $\left(\mathrm{kg} / \mathrm{m}^{3}\right), S$ is the fish's surface area $\left(\mathrm{m}^{2}\right)$, and $C_{\mathrm{D}}$ is a dimensionless drag coefficient (Webb 1975) of order 0.1 . A fish's instantaneous thrust or power, $P$ (in $\mathrm{kg} \cdot \mathrm{m}^{-2} \cdot \mathrm{s}^{-3}$ ), is given by the product of its drag, $D$, and relative velocity, $U_{\mathrm{r}}$,

$$
P=D U_{\mathrm{r}}
$$

and the integration of the power output through the swimming duration, $t$ (in s), equals the total work, $W$ $\left(\mathrm{kg} \cdot \mathrm{m}^{-2} \cdot \mathrm{s}^{-2}\right)$, done by a fish over its pathway:

$$
W=\int P d t .
$$

By combining Eqs. 1, 2, and 3 a general form for the work done by a fish over a migratory path is found:

$$
W=\frac{1}{2} \rho C_{\mathrm{D}} S \int U_{\mathrm{r}}^{3} d t .
$$

This assumes that the drag coefficient is a weak function of velocity and can be considered constant. This is very reasonable; Webb (1975) states $C_{\mathrm{D}} \sim U^{-0.2}$, and typical drag curves show constant drag coefficients at Reynolds numbers near $10^{6}$ (e.g., Tritton 2002:33) as is typical of large fish in large rivers.

For a discretized pathway, relative velocity is determined partly by the distance between discrete points along the fish's migration and the traversal time from point to point. This is combined with the groundreferenced velocity of the fluid flow and the relative angle between the two:

$$
U_{\mathrm{r}}=\frac{d}{t} \cos \theta+U
$$

where $d$ is the distance (in $\mathrm{m}$ ) between measured locations along a path, $U$ is the water velocity (in $\mathrm{m} / \mathrm{s}$ ) and $\theta$ is the angle between the fish path direction and the flow direction.

Eq. 4 comprises the set of parameters for a fish and its physical environment necessary to estimate absolute costs associated with distinct migratory pathways. Because it includes a representation of total drag (Eq. 1) including velocity (of the fish relative to the water through which it is swimming), and because a fish must match this drag in order to propel itself upstream, Eq. 4 
can be applied to the flow field in a river as a cost function for migration. There are three assumptions necessary to use Eq. 4 to estimate energetic costs for upstream migration. First, migration must be the dominant motivating factor in fish movement and pathway selection. Second, for any single fish during any measured traverse, these variables are assumed constant: density of water, cross-sectional area of the fish, and drag coefficient. None of these properties of the fish-river system should change over time scales short enough to be relevant to the fish's pathway-traversal process. Third, this formulation assumes that turbulent coherent structures play a minor role in overall energy requirements. The two main, possible sources of error from these assumptions are (1) use of velocity refugia (e.g., recirculation zones downstream of sand dunes; Webb et al. 1996 and Gerstner 1998) and (2) capture of vertical-axis vortices (Liao et al. 2003). We argue that flow refuging must be a small factor; fishes driven to migrate for spawning are likely to spend only a very small fraction of time holding a station because they must arrive at their spawning location in coordination with the completion of gamete development (de Gaudemar and Beall 1998). Vortex capture could however contribute a large source of error in cases where groups of fish are migrating together and vortices shed from leading fish can be captured by trailing fish. This framework is therefore limited to the pathways of solitary migrating fish.

It is currently not possible to incorporate these or other effects of turbulence using field-based data. These issues could only be resolved with very-high-resolution data. Essentially, one would need to track a fish and survey flow with data frequencies much greater than 1 Hz. This would require fixed-position hydrophones in order to maintain detailed positional measurements of the fish location as well as fixed-position acoustic Doppler devices to resolve coherent flow structures. However, because we are primarily interested in fish that migrate long distances, the cost and effort associated with collecting this type of information is highly prohibitive. Possibly, it could be a fruitful course of investigation in another setting.

In order to make Eq. 4 comparable between fish, i.e., similar fish should experience the relative expenditure between two pathways as approximately equivalent, Eq. 4 is normalized to the minimum amount of work, $W_{\min }$, necessary to maintain a position in the lowest mean current velocity, $U_{\min }$, found near a pathway's origin:

$$
f=\frac{W}{W_{\min }}=\frac{\frac{1}{2} \rho C_{\mathrm{D}} S \int U_{\mathrm{r}}^{3} d t}{\frac{1}{2} \rho C_{\mathrm{D}} S \int U_{\text {min }}^{3} d t}=\frac{\int U_{\mathrm{r}}^{3} d t}{U_{\text {min }}^{3} t} .
$$

Eq. 6 gives the final form of the cost function for a fish's migratory pathway relative to the effort that the fish would need to hold a local position. In essence this provides an exclusively physical cost function that is a representation of the environmental conditions that a fish would encounter along a particular course. Because the total time in transit must be held to the observed transit time, the cost associated with swimming across a channel is related to the relative velocity increases from Eq. 5. However, there is no specific cost associated with flow-normal movements, i.e., moving laterally. This cost-function is particularly useful because it can be calculated from physical surveys of river hydraulics alone and because a wide range of fishes in rivers satisfy the assumptions used to derive it. In the next section we show an example applying this theory to the upstream pathway of a migrating pallid sturgeon.

\section{Example Data Analysis: \\ Pallid Sturgeon in the Missouri River}

On 28 April 2010 a gravid female pallid sturgeon swam $1.8 \mathrm{~km}$ upstream in the Missouri River near Boonville, Missouri, USA, over the course of 7.5 hours (Fig. 1). During this portion of her migration (that totaled $50 \mathrm{~km}$ over three weeks in its entirety), the fish was located 16 times with telemetric transmitters that had been previously implanted. To relocate the fish, a boat equipped with directional hydrophones would maneuver until the sound from the transmitter reached maximum strength directly under the bow of the boat. Operators would record the time and the position of the boat on a differential global positioning system device with sub-meter accuracy. Also implanted in the fish was a data storage tag (DST) that recorded ambient pressure of the fish's environment at half-hour intervals. After spawning was completed around 1 May 2010, the fish was recaptured, the DST tag and its data were retrieved, and the fish was returned to the river.

While the fish was being relocated by the tracking boat, another boat equipped with an acoustic Doppler current profiler (ADCP) surveyed flow velocities in cross sections of the river that intersected locations where the fish had been found. For each relocation position, ADCP crosssection surveys were repeated four times in order to allow for averaging out the effects of variability in flow-velocity estimates. Total collection time for each set of crosssection lines took between 12 and 15 minutes. This duration combined with the number of replicate passes over each portion of the cross section struck a balance between enough measurements to create the average velocity field over a single transect and the ability to capture cross sections quickly enough to follow closely behind the fish. Velocity cross sections were surveyed within 15 to 20 minutes of relocating the fish.

Velocity surveys were sufficient to resolve detail at $0.25 \mathrm{~m}$ in the vertical dimension and $2.5 \mathrm{~m}$ in the horizontal dimension. The root-mean-square displacement of the ADCP from the planned survey lines over all cross sections is $2 \mathrm{~m}$. This positioning error is of the order of the size of the fish and is smaller than the planform resolution of the survey. Therefore it is appropriate to interpret the cross sections as localized surveys of the river's flow field. With an average width 

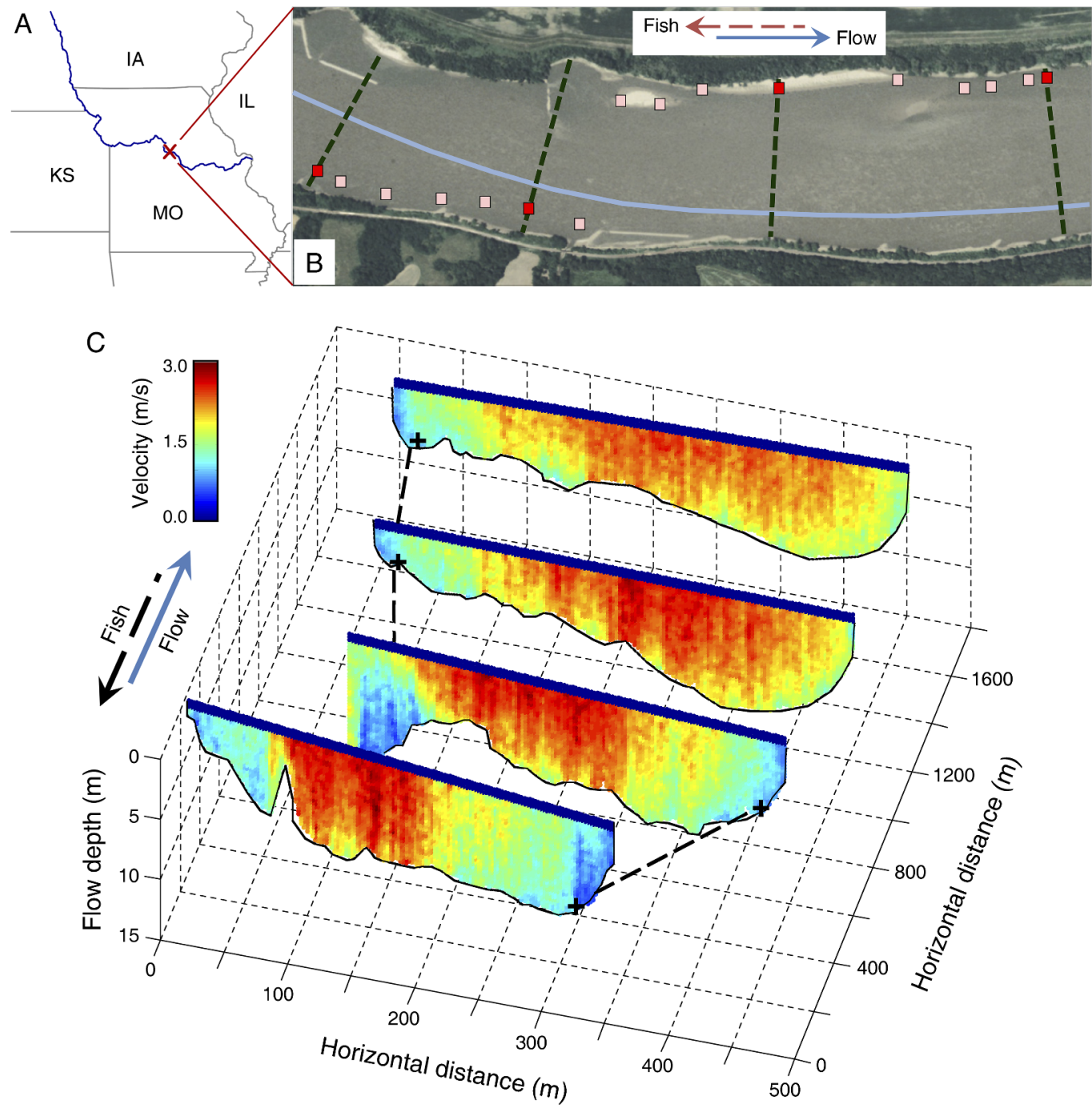

FIG. 1. (A) Regional map showing the Missouri River with the red "X" (the intersection of the two red lines) indicating the location of the surveyed migration pathway. (B) Aerial photo overlain with the approximate thalweg (blue line), and the fish relocation positions (pink squares). Acoustic Doppler current profiler (ADCP) transects were surveyed at every fish relocation position. Red squares and dashed lines indicate fish positions and ADCP transects plotted in panel C. Upstream is to the left on the photo. Overall, the fish traversed up the inside bank, crossed the thalweg, and proceeded up the next inside bank. (C) Oblique view of the migration pathway including a portion of the surveyed velocity field. Mean velocity is plotted in perspective view from the four ADCP transects (the four black dashed lines indicated in panel B). Flow is from the bottom to the top of panel C, and the sturgeon's path and telemetric location (black dashed lines and black crosses) is from the top of panel C to the bottom. This gives a sense of the three-dimensional velocity field that a fish must navigate. The blue-black band at the top of each cross section indicates those portions of the water column that cannot be sampled by our ADCP.

and depth of $\sim 400 \mathrm{~m}$ and $8 \mathrm{~m}$, respectively, the survey resolution equates to about 5000 independent average velocities per cross section. Because these data were collected at length scales larger than the size at which the fish is likely to be sensing its environment (Goodwin et al. 2006), it is appropriate to use the survey for populating a set of alternative paths through which the fish could pass.

DST data were downloaded and depths, $H$ (in m), were calculated from pressure measurements in the instruments native units, $p$ (in dbar; 1 decibar $=10 \mathrm{kPa})$.
With an estimate for gravitational acceleration, $g=9.80$ $\mathrm{m} / \mathrm{s}^{2}$, and water density, $\rho=1000 \mathrm{~kg} / \mathrm{m}^{3}$, the conversion from pressure to depth is $H=1.02 \mathrm{p}$. Because the DST logs pressure every 30 minutes, depth points were interpolated within the temporal domain at times when the fish was relocated. The result is a data set for the migratory pathway of the fish: (1) horizontal referencing, with Universal Transverse Mercator coordinates (UTM zone 15) in meters; (2) vertical referencing, with depth coordinates in meters; and (3) temporal coordinates, in seconds (Fig. 1). 
Fig. 1B shows a map of the fish's progress from east to west moving upstream on 28 April 2010. This section of the migratory pathway was surveyed in near real time as the fish traversed the inner bank of a bend, crossed the channel to the opposite inner bank and traversed the subsequent inner bank for the remainder of the survey day. The position where the fish crossed the channel is coincident with the location where the thalweg completes its crossing from the upstream outer bank to the downstream outer bank. Qualitatively, this suggests that the fish selected a path that avoided the relatively highvelocity thalweg in favor of lower velocity margins of the river. Avoiding the high-velocity thalweg results in a lower requirement of work necessary to swim through a reach, and by going from inner bank to inner bank on consecutive bends, the fish minimizes the sinuosity of its path, resulting in the shortest possible route from its initial downstream position to its migratory apex. This also requires the fish to cross the thalweg once per bend. Although this would seem costly, it is clearly less costly than continuing along a single bank and remaining in the thalweg along every other bend.

As the fish swam upstream, 16 consecutive cross sections were surveyed with the ADCP. Four of these cross sections are shown on the perspective view (Fig. 1C) of this bend to cross-over to bend river segment along with the route taken by the fish. The total distance of the path in this survey segment is $1800 \mathrm{~m}$. The average cross section spacing is $>100 \mathrm{~m}$, and it is assumed in the following analysis that this distance is appropriate to interpret each location as independent along the fish's path in the sense that the fish is capable of moving sufficiently vertically or laterally to choose any point as it moves from section to section. This is qualitatively and quantitatively corroborated by the spacing of the two points where the fish crossed the thalweg; the flow parallel distance between the points on either side of the thalweg crossing (Fig. 1B) is $\sim 65 \mathrm{~m}$ and the flow normal distance is $\sim 300 \mathrm{~m}$.

The relative energy cost of the pathway taken by the tracked sturgeon is estimated using Eq. 6 to be 210 (unitless). This can be interpreted as multiplicative factor; its path was 210 times more costly than holding position at the lowest velocity location at the downstream end of the reach $\left(U_{\mathrm{m}}=0.6 \mathrm{~m} / \mathrm{s}\right)$. In order to assess this energetic cost relative to the alternatives and the least-cost pathway, those energy costs must also be calculated.

For this particular example it is not feasible to uniquely define the least-cost path using Dijkstra's (1959) algorithm because it would require $\sim 5000^{16}$ calculations. Instead, we compare the actual path to the calculated costs of $10^{5}$ random three-dimensional migration pathways through the surveyed network (Fig. 2). The random pathways are defined by passing through one of the velocity measurements in each of the surveyed sections over the same duration of time as the actual pathway, thus creating a population of artificial,

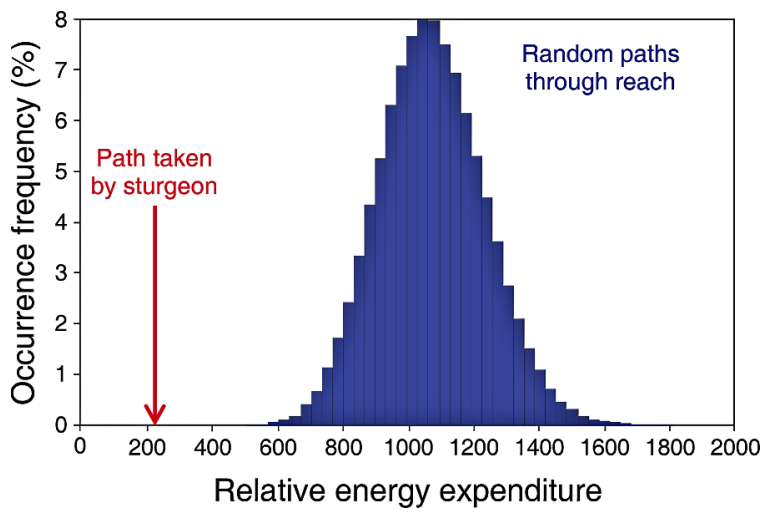

FIG. 2. Comparison of energy costs of random migration pathways through the reach compared to the cost of the pathway taken by the tracked sturgeon. These are obtained by applying Eq. 6 as a cost function to the velocity fields through the reach in Fig. 1B. The histogram shows the distribution of energy costs for $10^{5}$ random upstream-migration pathways with a mean $=1100$ and $\mathrm{SD}=200$ (unitless). The red arrow shows the cost of the sturgeon's pathway, $\sim 200$, which is less than $1 / 5$ of the mean cost of a random pathway.

possible pathways. The mean energetic cost of this distribution of pathways is 1100 with a standard deviation of 200. The minimum cost within the random pathway distribution is $\sim 400$. If one simply connected each of the minimum-velocity nodes through the flow network, the relative energetic cost would be 70 . Although this is a physically unattainable cost because it does not penalize for crossing the channel, it does set an interpretable limit on the energy costs of possible pathways; all pathways must be energetically more expensive than this minimum. Because the actualpathway cost is lower than the lowest random-pathway cost and because the actual-pathway cost is much closer to the lower limit than it is to the mean cost of the random-pathway distribution, we contend that the fish selected a pathway to minimize its energy expenditure during migration.

\section{Discussion}

The above example demonstrates the application of the cost-function derived in Example data analysis,,,, (above), to field data and how those data are collected and analyzed. In order to use these kinds of data to statistically test the pathway-optimization hypothesis or other similar hypotheses, many more equivalent data sets would be needed. A number of issues about the data stratification would also have to be addressed in such a test. These include (1) How many different individual fish would be sufficient? (2) How many unique bends or thalweg cross-overs would be needed? (3) How many or what density of flow-velocity surveys would be necessary? And (4) How many distinct discharges would need to be sampled? For the example presented using pallid sturgeon, much more effort will be needed in coming years to fully test the migration pathway optimization 
hypothesis. However, the methodology presented offers a coherent manner in which to measure the relative energy cost of migration in field settings using standard fish tracking methods coupled with acoustic Doppler based flow-field surveys.

Although we do not have conclusive hypothesis test results, the implications of this test are clear nonetheless. If fishes indeed optimize their relative allocations of resources for migration and reproductive needs, then this type of analysis could offer insight into alternatives for management strategies of large-river fishes. This assumes that cues exist for fishes to migrate and to spawn and does not address the many other needs of fishes migrating to spawn. Given these caveats, and in order to support reproduction, it could be a beneficial management tool in cases where it can be demonstrated that fishes minimize the energy requirements for migration. Many large rivers (Missouri, Mississippi, Ohio, Danube, Volga, Rhine, and so forth) have been modified for navigation and bank stabilization by construction of rock revetments and rock wing dikes (groins). Considering low-velocity fish-migration corridors when designing new or rehabilitated river-training structures could benefit greater overall reproductive potential of managed fish populations that require longdistance spawning migrations.

\section{ACKNOWLEDGMENTS}

This work is partly funded by the U.S. Geological Survey and the U.S. Army Corps of Engineers Missouri River Recovery Program. Many thanks to the entire field crew for their dedicated work during the telemetry effort, to David Galat and Tim Counihan for early reviews and suggestions, and to two anonymous reviewers.

\section{Literature Cited}

Ahuja, R. K., T. L. Magnanti, and J. B. Orlin. 1993. Network flows: theory, algorithms, and applications. Prentice Hall, Englewood Cliffs, New Jersey, USA.

Anderson, E., W. McGillis, and M. Grosenbaugh. 2001. The boundary layer of swimming fish. Journal of Experimental Biology 204:81-102.

Boisclair, D., and M. Tang. 1993. Empirical analysis of the influence of swimming pattern on the net energetic cost of swimming in fishes. Journal of Fish Biology 42:169-183.

Brandimarte, P., and G. Zotteri. 2007. Introduction to distribution logistics. Wiley-Interscience, Hoboken, New Jersey, USA.

Colavecchia, M., C. Katopodis, R. Goosney, D. Scruton, and R. McKinley. 1998. Measurement of burst swimming performance in wild Atlantic salmon (Salmo salar L.) using digital telemetry. Regulated Rivers 14:41-51.

Crossin, G., S. Hinch, A. Farrell, D. Higgs, A. Lotto, J. Oakes, and M. Healey. 2004. Energetics and morphology of sockeye salmon: effects of upriver migratory distance and elevation. Journal of Fish Biology 65:788-810

de Gaudemar, B., and E. Beall. 1998. Effects of overripening on spawning behaviour and reproductive success of Atlantic salmon females spawning in a controlled flow channel. Journal of Fish Biology 53:434-446.
DeLonay, A. J., R. B. Jacobson, D. M. Papoulias, D. G. Simpkins, M. L. Wildhaber, J. M. Reuter, T. W. Bonnot, K. A. Chojnacki, G. E. Mestl, and M. J. Mac. 2009. Ecological requirements for pallid sturgeon reproduction and recruitment in the Lower Missouri River: a research synthesis 2005-08. U.S. Geological Survey Scientific Investigations Report 2009-5201.

DeLonay, A. J., R. B. Jacobson, D. M. Papoulias, M. L. Wildhaber, K. A. Chojnacki, E. K. Pherigo, C. L. Bergthold, and G. E. Mestl. 2010. Ecological requirements for pallid sturgeon reproduction and recruitment in the Lower Missouri River. Annual report 2009. U.S. Geological Survey Open-File Report 2010-1215.

Dijkstra, E. 1959. A note on two problems in connection with graphs. Numerische Mathematik 1:317-322.

Drucker, E., and G. Lauder. 2002. Experimental hydrodynamics of fish locomotion: functional insights from wake visualization. Integrative and Comparative Biology 42:243257.

Gerstner, C. L. 1998. Use of substratum ripples for flow refuging by Atlantic cod, Gadus morhua. Environmental Biology of Fishes 51:455-460.

Goodman, R. A., J. M. Nestler, J. J. Anderson, L. I. Weber, and D. P. Loucks. 2006. Forecasting 3-D fish movement behavior using a Eulerian-Langrangian-agent method (ELAM). Ecological Modelling 192:197-223.

Hinch, S., and P. Rand. 2000. Optimal swimming speeds and forward-assisted propulsion: energy-conserving behaviours of upriver-migrating adult salmon. Canadian Journal of Fisheries and Aquatic Sciences 57:2470-2478.

Huitema, C. 2000. Routing in the Internet. Prentice Hall, Upper Saddle River, New Jersey, USA.

Jacobson, R., and D. Galat. 2006. Flow and form in rehabilitation of large-river ecosystems: an example from the Lower Missouri River. Geomorphology 77:249-269.

Jonsson, B., and N. Jonsson. 2006. Life-history effects of migratory costs in anadromous brown trout. Journal of Fish Biology 69:860-869.

Kinnison, M., M. Unwin, A. Hendry, and T. Quinn. 2001. Migratory costs and the evolution of egg size and number in introduced and indigenous salmon populations. Evolution 55:1656-1667.

Kinnison, M., M. Unwin, and T. Quinn. 2003. Migratory costs and contemporary evolution of reproductive allocation in male chinook salmon. Journal of Evolutionary Biology 16:1257-1269.

Liao, J. C., D. N. Beal, G. V. Landers, and M. S. Triantafyllou. 2003. Fish exploiting vortices decrease muscle activity. Science 302:1566-1569

Mayden, R., and B. Kuhajda. 1997. Threatened fishes of the world: Scaphirhynchus albus (Forbes \& Richardson, 1905) (Acipenseridae). Environmental Biology of Fishes 48:420421.

Rees, W. 2004. Least-cost paths in mountainous terrain. Computers and Geosciences 30:203-209.

Stoll, H. 1989. Least-cost electric utility planning. WileyInterscience, New York, New York, USA.

Tritton, D. J. 2002. Physical fluid dynamics. Clarendon, Oxford, UK

Webb, P. 1975. Hydrodynamics and energetics of fish propulsion. Bulletin of the Fisheries Research Board of Canada 190.

Webb, P., C. Gerstner, and S. Minton. 1996. Station-holding by mottled sculpin, Cottus bairdi (Teleostei: Cottidae), and other fishes. Copeia 1996:488-493.

Weihs, D. 1974. Energetic advantages of burst swimming of fish. Journal of Theoretical Biology 48:215-229. 